\title{
Implementation of Reduced Form Mechanisms: A Simple Approach and a New Characterization*
}

\author{
Sergiu Hart ${ }^{\dagger} \quad$ Philip J. Reny ${ }^{\ddagger}$
}

December 21, 2011

\begin{abstract}
We provide a new characterization of implementability of reduced form mechanisms in terms of straightforward second-order stochastic dominance. In addition, we present a simple proof of Matthews' (1984) conjecture, proved by Border (1991), on implementability.
\end{abstract}

\section{Introduction}

In mechanism design contexts, such as those with type-dependent outside options, it is sometimes more natural and convenient to optimize over reduced form mechanisms - i.e., interim probability assignments and cost functionsrather than the underlying mechanisms themselves. ${ }^{1}$ Reduced form mechanisms also play a useful role in the literature on the equivalence between Bayesian and dominant strategy implementation. ${ }^{2}$ In contexts such as these,

*First version: August 2010. Research partially supported by the National Science Foundation (SES-0922535) and the European Research Council (FP7-249159). The authors thank Benny Moldovanu, Yosi Rinott, Marco Scarsini, and Benjy Weiss, for useful discussions and suggestions.

${ }^{\dagger}$ Department of Economics, Institute of Mathematics, and Center for the Study of Rationality, The Hebrew University of Jerusalem. E-mail: hart@huji.ac.il Web site: http://www.ma.huji.ac.il/hart

${ }_{\ddagger}$ Department of Economics, University of Chicago. E-mail: preny@uchicago.edu Web site: http://home.uchicago.edu/ preny

${ }^{1}$ See, e.g., Mierendorff (2009).

${ }^{2}$ See, e.g., Manelli and Vincent (2010) and Gershkov, Moldovanu, and Shi (2011). 
it is important to know when a reduced form mechanism can actually be implemented. Maskin and Riley (1984) posed and studied this question, and obtained a partial solution. Matthews (1984) made further progress and in addition conjectured that an intuitive inequality constraint is necessary and sufficient for implementability. Border (1991) finally solved the problem by proving Matthews' conjecture.

The purpose of the present note is twofold. First, we provide an alternative characterization of implementability in terms of straightforward second-order stochastic dominance, ${ }^{3}$ and second, we offer a simpler, more direct proof of Matthews' conjecture. Our work, like all of the work mentioned above, focuses on the classic mechanism design setting in which there is a single indivisible object to be allocated to one of $n$ ex-ante symmetric agents, each of whom has quasi-linear utility and whose private information, which may be quite general, is drawn independently from a common distribution. ${ }^{4}$

\section{Results}

The underlying probability space (the "type space") is ${ }^{5}(T, \mathcal{T}, \lambda)$. The number of agents is $n$. Because the implementability question relates only to a mechanism's probability assignment function, we define a mechanism here in these terms only. ${ }^{6}$ Thus, a mechanism consists of $n$ functions $q_{1}, q_{2}, \ldots, q_{n}$, with $q_{i}: T^{n} \rightarrow[0,1]$ for each $i=1,2, \ldots, n$, such that $\sum_{i=1}^{n} q_{i}\left(t_{1}, t_{2}, \ldots, t_{n}\right) \leq 1$ for every $t_{1}, t_{2}, \ldots, t_{n}$ in $T$; here $q_{i}\left(t_{1}, t_{2}, \ldots, t_{n}\right)$ is the probability that agent $i$ gets the object when the reported types are $t_{1}, t_{2}, \ldots, t_{n}$. A mechanism is symmetric if $q_{i}\left(t_{\pi(1)}, t_{\pi(2)}, \ldots, t_{\pi(n)}\right)=q_{\pi(i)}\left(t_{1}, t_{2}, \ldots, t_{n}\right)$ for every permutation $\pi$ of $\{1,2, \ldots, n\}$ and every agent $i=1,2, \ldots, n$; i.e., the "names" $(1,2, \ldots, n)$ of the agents do not matter. A symmetric mechanism is thus given by a function $q \equiv q_{1}: T^{n} \rightarrow[0,1]$ such that $q\left(t_{1}, t_{2}, \ldots, t_{n}\right)$ is invari-

\footnotetext{
${ }^{3}$ Which may be useful when optimizing; see Remark (c) below.

${ }^{4}$ For the case of asymmetric agents with finite type spaces, see Border (2007).

${ }^{5}$ There are no requirements on the probability space. All functions will be assumed measurable, and all statements to hold almost surely.

${ }^{6}$ Thus the cost function as well as additional constraints (such as participation constraints), which are not important for our purposes, are left unspecified.
} 
ant to permutations of $\left(t_{2}, \ldots, t_{n}\right)$ and $\sum_{i} q_{i} \leq 1$, where $q_{i}\left(t_{1}, t_{2}, \ldots, t_{n}\right):=$ $q\left(t_{i}, t_{2}, \ldots, t_{i-1}, t_{1}, t_{i+1}, \ldots, t_{n}\right)$ (i.e., interchange the first and the $i$ th coordinates); $q\left(t_{1}, t_{2}, \ldots, t_{n}\right)$ is the probability that an agent whose type is $t_{1}$ gets the object when the other agents are of types $t_{2}, \ldots, t_{n}$.

A reduced form is a mapping $Q: T \rightarrow[0,1]$. A reduced form $Q$ is implementable if there exists a symmetric mechanism given by $q: T^{n} \rightarrow[0,1]$ such that $Q(t)=\int_{T^{n-1}} q\left(t, t_{2}, \ldots, t_{n}\right) \mathrm{d} \lambda\left(t_{2}\right) \cdots \mathrm{d} \lambda\left(t_{n}\right)$ for all $t \in T$; i.e., $Q(t)$ is the overall probability that an agent of type $t$ gets the object. In this case we say that $Q$ is the reduced form of $q$, or that $q$ generates $Q$. Our concern is whether a given reduced form $Q$ is implementable. ${ }^{7}$

As will be shown, an important special case consists of the type space $\left([0,1], \mathcal{B}, \lambda^{*}\right)$, with $\lambda^{*}$ the Lebesgue measure, and $q^{*}\left(t_{1}, t_{2}, \ldots, t_{n}\right)=1$ if $t_{1}>$ $\max \left\{t_{2}, \ldots, t_{n}\right\}$, and $q^{*}\left(t_{1}, t_{2}, \ldots, t_{n}\right)=0$ otherwise. That is, the agents' types are each uniformly distributed on $[0,1]$ and the mechanism gives the object to the agent whose type is highest. Its reduced form is easily seen to be $Q^{*}(t)=t^{n-1}$ for all $t \in[0,1]$ (when $n=2$, the distribution of $Q^{*}$ is uniform on $[0,1])$.

Theorem 1 The following conditions on $Q: T \rightarrow[0,1]$ are equivalent:

(i) $Q$ is implementable; i.e., it is the reduced form of a symmetric mechanism.

(ii) $Q$ satisfies, for every ${ }^{8} \alpha \in[0,1]$,

$$
\int_{[Q>\alpha]} Q(t) \mathrm{d} \lambda(t) \leq \frac{1}{n}-\frac{1}{n}(\lambda[Q \leq \alpha])^{n}
$$

(iii) $-Q$ second-order stochastically dominates $-Q^{*}$.

\footnotetext{
${ }^{7}$ The symmetry requirement here is somewhat more demanding than the one in Maskin and Riley (1984) and Border (1991), who do not require the invariance of $q\left(t_{1}, t_{2}, \ldots, t_{n}\right)$ with respect to permutations of $\left(t_{2}, \ldots, t_{n}\right)$. Ours is however the natural symmetry requirement when all agents are symmetric, and in particular it does not affect the implementability of a reduced form $Q$, since averaging all $n$ ! permutations of a mechanism-i.e., $\tilde{q}_{i}\left(t_{1}, \ldots, t_{n}\right):=(1 / n !) \sum_{\pi} q_{\pi^{-1}(i)}\left(t_{\pi(1)}, t_{\pi(2)}, \ldots, t_{\pi(n)}\right)$-yields a symmetric mechanism in our sense.

${ }^{8}[Q<\alpha]$ is short for $\{t \in T: Q(t)<\alpha\}$; similarly for the other events.
} 


\section{Remarks.}

(a) Condition (ii) is due to Matthews (1984); ${ }^{9}$ Border (1991) shows the equivalence of (i) and (ii); condition (iii) is new.

(b) Condition (iii) means that for every increasing and concave function $u:[0,1] \rightarrow \mathbb{R}$ we have ${ }^{10} \mathbf{E}[u(-Q)] \geq \mathbf{E}\left[u\left(-Q^{*}\right)\right]$; equivalently, for every increasing and convex function $v($ take $u(x)=-v(-x)$ ), we have $\mathbf{E}[v(Q)] \leq$ $\mathbf{E}\left[v\left(Q^{*}\right)\right]$, or

$$
\int_{T} v(Q(t)) \mathrm{d} \lambda(t) \leq \int_{T^{*}} v\left(Q^{*}(t)\right) \mathrm{d} \lambda^{*}(t)=\int_{0}^{1} v\left(t^{n-1}\right) \mathrm{d} t .
$$

In terms of distributions, this amounts to $Q^{*}$ being obtained from $Q$ by increasing values (pointwise) and applying mean-preserving spreads (see Hadar and Russell 1969, Hanoch and Levy 1969, Rothschild and Stiglitz 1970, and the book of Shaked and Shanthikumar 2010).

Equivalently, there exists a probability space $(\Omega, \mathcal{F}, \mathbf{P})$ and two random variables $X$ and $Y$ defined on it, such that $X$ and $Q$ have the same distribution, $Y$ and $Q^{*}$ have the same distribution, and ${ }^{11} \mathbf{E}[Y \mid X] \geq X$; this construct is known as coupling (see Strassen 1965, Theorem 9; Machina and Pratt 1997, Theorem 3'; Shaked and Shanthikumar 2010, Theorem 4.A.5).

(c) An immediate consequence of (iii) and (2) is that, for each increasing and convex function $v$, the maximum of $\mathbf{E}[v(Q)]$ over all implementable $Q$ is attained at $Q^{*}$ and equals $\int_{0}^{1} v\left(t^{n-1}\right) \mathrm{d} t$.

\footnotetext{
${ }^{9}$ The fact that it suffices to consider the inequality (1) only on sets of the form $[Q>\alpha]$, rather than on all measurable sets as in Matthews (1984), is immediate (see Proposition 3.2 of Border 1991).

${ }^{10} \mathbf{E}$ denotes expectation (with respect to the appropriate probability measure: $\lambda$ for $Q$, and $\lambda^{*}$ for $\left.Q^{*}\right)$.

${ }^{11}$ I.e., $\mathbf{P}[X \leq \alpha]=\lambda[Q \leq \alpha]$ and $\mathbf{P}[Y \leq \alpha]=\lambda^{*}\left[Q^{*} \leq \alpha\right]=\alpha^{1 /(n-1)}$ for every $\alpha \in$ $[0,1]$. The change from $X$ to $Y$ can be understood as increasing values pointwise (from $X$ to $\mathbf{E}[Y \mid X]$ ) and applying mean-preserving spreads (from $\mathbf{E}[Y \mid X]$ to $Y$ ).
} 


\section{Proof of Theorem 1. ${ }^{12}$}

- (i) implies (ii). As in Matthews (1984) and Border (1991, Lemma 5.1), using symmetry and $\sum_{i} q_{i} \leq 1$ yields for any measurable $A \subset T$, in particular for ${ }^{13} A=[Q>\alpha]$,

$$
\begin{aligned}
n \mathbf{E}\left[Q(t) \mathbf{1}_{[t \in A]}\right] & =\mathbf{E}\left[\sum_{i=1}^{n} Q\left(t_{i}\right) \mathbf{1}_{\left[t_{i} \in A\right]}\right]=\mathbf{E}\left[\sum_{i=1}^{n} q_{i}\left(t_{1}, \ldots, t_{n}\right) \mathbf{1}_{\left[t_{i} \in A\right]}\right] \\
& \leq \mathbf{E}\left[\mathbf{1}_{\cup_{i}\left[t_{i} \in A\right]}\right]=1-(\lambda[T \backslash A])^{n} .
\end{aligned}
$$

- (ii) implies (iii). Put $p:=\lambda[Q \leq \alpha]$; we have ${ }^{14}$

$$
\begin{aligned}
\int_{T}[Q(t)-\alpha]_{+} \mathrm{d} \lambda(t) & =\int_{[Q>\alpha]} Q(t) \mathrm{d} \lambda(t)-\alpha(1-p) \leq \frac{1}{n}-\frac{1}{n} p^{n}-\alpha+\alpha p \\
& \leq \frac{1}{n}-\alpha+\frac{n-1}{n} \alpha^{\frac{n}{n-1}}=\int_{0}^{1}\left[t^{n-1}-\alpha\right]_{+} \mathrm{d} t \\
& =\int_{T^{*}}\left[Q^{*}(t)-\alpha\right]_{+} \mathrm{d} \lambda^{*}(t),
\end{aligned}
$$

where the first inequality is (1), and the second is the classical Young's inequality ${ }^{15} \alpha p \leq(1 / n) p^{n}+((n-1) / n) \alpha^{n /(n-1)}$. Hence $\int_{T}[Q(t)-\alpha]_{+} \mathrm{d} \lambda(t) \leq$ $\int_{T^{*}}\left[Q^{*}(t)-\alpha\right]_{+} \mathrm{d} \lambda^{*}(t)$ for all $\alpha \in[0,1]$, which is equivalent to $(2)$ since every increasing convex function $v$ (with $v(0)=0$, which does not affect (2)) lies in the closed convex cone generated by the functions $\phi_{\alpha}(x):=[x-\alpha]_{+}$for all $\alpha \in[0,1] .{ }^{16}$

- (iii) implies (i). Assume that $-Q$ second-order stochastically dominates $-Q^{*}$. Applying coupling (see Remark (b)) yields a probability space

\footnotetext{
${ }^{12}$ Showing that conditions (ii) and (iii) are each necessary for the implementability of $Q$ is quite straightforward; the difficulty lies in proving that these conditions are sufficient (cf. "(iii) implies (i)").

${ }^{13} \mathbf{1}_{W}$ denotes the indicator of the event $W$.

${ }^{14}[x]_{+}:=\max \{x, 0\}$.

${ }^{15}$ Which follows, for instance, from the concavity of log (after applying log to both sides).

${ }^{16}$ While for each $\alpha$ the inequality in (3), which can be written as $\int_{[Q>\alpha]} Q(t) \mathrm{d} \lambda(t) \leq$ $1 / n+((n-1) / n) \alpha^{n /(n-1)}-\alpha \lambda[Q \leq \alpha]$, is strictly weaker than inequality (1), our result implies that "(3) for all $\alpha$ " is equivalent to "(1) for all $\alpha$ " (this equivalence can also be proved quite directly). Of course, our purpose here is to provide a simple and self-contained proof of the equivalence of (i)-(iii).
} 
and two random variables $X$ and $Y$ defined on it, such that $X$ and $Q$ have the same distribution, $Y$ and $Q^{*}$ have the same distribution, and $\mathbf{E}[Y \mid X] \geq X$.

Let $\left(X_{i}, Y_{i}\right)$, for $i=1,2, \ldots, n$, be $n$ independent pairs of random variables, all identically distributed and with the same distribution as the pair $(X, Y)$. For each $\left(t_{1}, t_{2}, \ldots, t_{n}\right) \in T^{n}$ define the event

$$
A\left(t_{1}, t_{2}, \ldots, t_{n}\right):=\left[X_{1}=Q\left(t_{1}\right), X_{2}=Q\left(t_{2}\right), \ldots, X_{n}=Q\left(t_{n}\right)\right],
$$

and put $^{17}$

$$
\tilde{q}\left(t_{1}, t_{2}, \ldots, t_{n}\right):=\mathbf{P}\left[Y_{1}>\max _{j \neq 1} Y_{j} \mid A\left(t_{1}, t_{2}, \ldots, t_{n}\right)\right]
$$

Then $\sum_{i} \tilde{q}_{i}\left(t_{1}, \ldots, t_{n}\right)=\sum_{i} \mathbf{P}\left[Y_{i}>\max _{j \neq i} Y_{j} \mid A\left(t_{1}, \ldots, t_{n}\right)\right] \leq 1$ (these $n$ events are disjoint), and so $\tilde{q}: T^{n} \rightarrow[0,1]$ yields a symmetric mechanism. Moreover, integrating over $\left(t_{2}, \ldots, t_{n}\right) \in T^{n-1}$ (recall that $\left(Q\left(t_{2}\right), \ldots, Q\left(t_{n}\right)\right)$ and $\left(X_{2}, \ldots, X_{n}\right)$ have the same distribution) gives the reduced form $\tilde{Q}$ of $\tilde{q}$ :

$$
\tilde{Q}\left(t_{1}\right)=\mathbf{P}\left[Y_{1}>\max _{j \neq 1} Y_{j} \mid X_{1}=Q\left(t_{1}\right)\right]
$$

Now $\mathbf{P}\left[Y_{j} \leq y\right]=\lambda^{*}\left[Q^{*} \leq y\right]=y^{1 /(n-1)}$ for every $y \in[0,1]$ (since $Y_{j}$ has the same distribution as $\left.Q^{*}\right)$, which implies that $\mathbf{P}\left[\max _{j \neq 1} Y_{j} \leq y \mid X_{1}\right]=$ $\prod_{j=2}^{n} \mathbf{P}\left[Y_{j} \leq y\right]=\left(y^{1 /(n-1)}\right)^{n-1}=y$ (we have used here the independence over $j$ ). Thus $\max _{j \neq 1} Y_{j}$ is uniformly distributed on $[0,1]$, and moreover independent of $X_{1}$; hence (4) yields

$$
\tilde{Q}\left(t_{1}\right)=\int_{0}^{1} \mathbf{P}\left[Y_{1}>y \mid X_{1}=Q\left(t_{1}\right)\right] \mathrm{d} y=\mathbf{E}\left[Y_{1} \mid X_{1}=Q\left(t_{1}\right)\right] \geq Q\left(t_{1}\right)
$$

(recall that $\mathbf{E}\left[Y_{1} \mid X_{1}\right] \geq X_{1}$ ). It only remains to rescale: put

$$
q\left(t_{1}, \ldots, t_{n}\right):=\tilde{q}\left(t_{1}, \ldots, t_{n}\right) \frac{Q\left(t_{1}\right)}{\tilde{Q}\left(t_{1}\right)}
$$

\footnotetext{
${ }^{17}$ We write $\mathbf{P}$ for the probability measure on the space on which all $X_{i}$ and $Y_{i}$ are defined.
} 
(where we take $0 \cdot 0 / 0$ as 0 ); then $q$ yields a symmetric mechanism (since $q \leq \tilde{q}$ ), and its reduced form is precisely the given $Q$.

Finally, consider symmetric mechanisms that are maximal, in the sense that $\sum_{i=1}^{n} q_{i}=1$. If $Q$ is the reduced form, then $\mathbf{E}\left[\sum_{i} q_{i}\right]=n \mathbf{E}[Q]$. It follows that an implementable reduced form $Q$ is the reduced form of a maximal mechanism if and only if $\mathbf{E}[Q]=1 / n$; in this case we will also call $Q$ maximal. Clearly, for any implementable $Q$ there is a maximal implementable $\tilde{Q}$ with $\tilde{Q}(t) \geq Q(t)$ for all ${ }^{18} t$. We have:

Corollary 2 The following conditions on $Q: T \rightarrow[0,1]$ with $\mathbf{E}[Q]=1 / n$ are equivalent:

(i-Max) $Q$ is the reduced form of a maximal symmetric mechanism.

(iii-Max) $Q$ second-order stochastically dominates $Q^{*}$.

Proof. $\mathbf{E}[Q]=1 / n$ implies that $\mathbf{E}[Q]=\mathbf{E}\left[Q^{*}\right]$ (since $Q^{*}$ is maximal), and in this case condition (iii) is equivalent to (iii-Max): indeed, for the coupled random variables $X$ and $Y$ of Remark (b), when $\mathbf{E}[X]=\mathbf{E}[Y]$ the conditions $\mathbf{E}[Y \mid X] \geq X, \mathbf{E}[Y \mid X]=X$, and $\mathbf{E}[Y \mid X] \leq X$, are all equivalent. ${ }^{19}$

Thus, $Q^{*}$ is obtained from an implementable maximal $Q$ by mean-preserving spreads; that is, $Q^{*}$ has the same distribution as $Q+Z$ for some "noise" $Z$ that is uncorrelated with $Q$ (i.e., $\mathbf{E}[Z \mid Q]=0$ ).

\section{References}

Border, K. C. (1991), "Implementation of Reduced Form Auctions: A Geometric Approach," Econometrica 59, 1175-1187.

Border, K. C. (2007), "Reduced Form Auctions Revisited," Economic Theory $31,167-181$

\footnotetext{
${ }^{18}$ If $Q$ is the reduced form of $q$, then let $\tilde{Q}$ be the reduced form of $\tilde{q}$, which is defined by $\tilde{q}:=q / \sum_{i} q_{i}$ (if $q_{i}=0$ for all i then take $\left.\tilde{q}:=1 / n\right)$.

${ }^{19}$ Informally: when the expectations are equal one cannot increase the values, and only mean-preserving spreads can be used.
} 
Gershkov, A., B. Moldovanu, and A. Shi (2011), "Bayesian and Dominant Strategy Implementation Revisited," University of Bonn, unpublished working paper.

Hadar, J. and W. Russell (1969), "Rules for Ordering Uncertain Prospects," American Economic Review 59, 25-34.

Hanoch, G. and H. Levy (1969), "The Efficiency Analysis of Choices Involving Risk," Review of Economic Studies 36, 335-346.

Machina, M. and J. Pratt (1997), "Increasing Risk: Some Direct Constructions," Journal of Risk and Uncertainty 14, 103-127.

Manelli, A., and D. Vincent (2010), "Bayesian and Dominant Strategy Implementation in the Independent, Private Values Model," Econometrica 78, 1905-1939.

Maskin, E. S. and J. Riley (1984), "Optimal Auctions with Risk-Averse Buyers," Econometrica 52, 1473-1518.

Matthews, S. A. (1984), "On the Implementability of Reduced Form Auctions," Econometrica 52, 1519-1522.

Mierendorff, K. (2009), "Optimal Dynamic Mechanism Design with Deadlines, University of Bonn, unpublished working paper.

Rothschild, M. and J. Stiglitz (1970), "Increasing Risk: I. A Definition," Journal of Economic Theory 2, 225-243.

Shaked, M. and J. G. Shanthikumar (2010), Stochastic Orders, Springer.

Strassen, V. (1965), "The Existence of Probability Measures with Given Marginals," The Annals of Mathematical Statistics 36, 423-439. 